\title{
A Standardized, Structured Approach to Identifying Drug-Related Problems in the Intensive Care Unit: FASTHUG-MAIDENS
}

\author{
Vincent H Mabasa, Douglas L Malyuk, Elisa-Marie Weatherby, and Alice Chan
}

\section{INTRODUCTION}

$\mathrm{P}$ harmacy practice in the intensive care unit (ICU) is complex, because of the high acuity of patients' conditions and the large number of medications prescribed. Therefore, many pharmacists, especially those not familiar with ICU care, may feel overwhelmed and apprehensive in this setting. There is currently no standardized, structured approach to help pharmacists provide pharmaceutical care in the ICU. For that reason, routine tasks such as identifying drug-related problems are much harder to perform, and drug therapy is not always optimized.

In 2005, the FASTHUG mnemonic was proposed as a standardized approach to help ICU physicians ensure that all essential aspects of care for critically ill patients are met ${ }^{1}$. Implementation of the FASTHUG approach in a surgical ICU was subsequently shown to decrease the rates of ventilator-associated pneumonia. ${ }^{2}$ Although the mnemonic has been generally well received, some clinicians have modified it to better augment their particular ICU practices. ${ }^{3}$ Notably, the FASTHUG mnemonic was not designed to identify drug-related problems commonly seen in the ICU. Therefore, we developed a modified mnemonic, FASTHUG-MAIDENS, as a standardized, structured approach to identifying drug-related problems in the ICU (Table 1).

\section{DESCRIPTION OF FASTHUG-MAIDENS}

\section{$F$ is for Feeding}

Patients in the ICU may receive nutrition by a variety of methods, such as parenteral nutrition, tube feeding, sips of fluids, diet as tolerated, and no oral intake. This variety means there are several opportunities to optimize drug therapy, such as changing medications to the oral route from the parenteral route or vice versa, depending on how the patient is fed. For sustained-release medications administered in a form that cannot be crushed, an alternative dosage form, such as a liquid formulation or an immediate-release form, could be recommended to achieve equivalent total daily doses. If the patient is not tolerating enteral feeding and is experiencing high gastric residual volumes, the pharmacist should assess whether the problem is related to a medication. Prokinetic agents such as metoclopramide or erythromycin may be considered in these situations. The pharmacist should also assess the possibility of an interaction between a drug and a nutritional formulation administered by feeding tube that might affect absorption of the drug. For patients receiving parenteral nutrition, the pharmacist can monitor various laboratory parameters and suggest modification of the ingredients as appropriate.

\section{A is for Analgesia}

Patients in the ICU often require analgesia to treat various sources of pain, such as trauma, surgery, or other pre-existing medical conditions. Pain can be readily assessed with a pain scale, and the patient's level of pain should be checked routinely. ${ }^{4}$ Ideally, a patient will receive an amount of analgesic that is adequate (so that pain is not an issue) but not excessive (so that unwanted sedation and respiratory depression are avoided and to allow successful weaning from the ventilator)..$^{5}$ In addition, the pharmacist can assess the patient's situation and make suggestions about the most appropriate method for delivering analgesic medications, such as infusions, regularly scheduled intermittent doses, or longer-acting forms combined with as-needed doses.

\section{$\mathbf{S}$ is for Sedation}

Many of the issues discussed above for analgesia also apply to sedation. It is important for pharmacists to ensure that the appropriate sedative medications are being used when indicated. The most common type of sedative used in the ICU is benzodiazepines, including drugs such as midazolam and 
Table 1. The FASTHUG-MAIDENS Mnemonic

\begin{tabular}{llll} 
Letter & \multicolumn{1}{c}{ Definition } & Letter & \multicolumn{1}{c}{ Definition } \\
\hline $\mathrm{F}$ & Feeding & $\mathrm{M}$ & Medication reconciliation \\
\hline $\mathrm{A}$ & Analgesia & $\mathrm{A}$ & Antibiotics or anti-infectives \\
\hline $\mathrm{S}$ & Sedation & I & Indications for medications \\
\hline $\mathrm{T}$ & Thromboprophylaxis & $\mathrm{D}$ & Drug dosing \\
\hline $\mathrm{H}$ & Hyperactive or hypoactive delirium* & $\mathrm{E}$ & $\begin{array}{c}\text { Electrolytes, hematology, and other } \\
\text { laboratory results }\end{array}$ \\
\hline $\mathrm{U}$ & Stress ulcer prophylaxis & $\mathrm{N}$ & $\begin{array}{c}\text { No drug interactions, allergies, } \\
\text { duplications, side effects }\end{array}$ \\
\hline $\mathrm{G}$ & Glucose control & S & Stop dates \\
\hline
\end{tabular}

*In the original version of the FASTHUG mnemonic, $\mathrm{H}$ was for "head of the bed elevated".

lorazepam, but there may be times when a sedative-hypnotic agent would be ideal. The pharmacist should be involved in the decision to initiate, discontinue, and adjust doses of sedative medications, according to the clinical situation and the patient's score on a sedation scale. ${ }^{4}$ For example, propofol may be suitable if the patient requires only short-term sedation. ${ }^{5}$ However, benzodiazepines may be more appropriate if longer-term sedation is needed. ${ }^{6}$ The pharmacist should assess each patient daily to determine whether he or she would benefit from sedation therapy as a continuous infusion, as an intermittent dosing regimen, or on an as-needed basis.

\section{$\mathrm{T}$ is for Thromboembolic prophylaxis}

Almost all patients in the ICU should receive some form of thromboembolic prophylaxis.? However, some critically ill patients may not be receiving chemical thromboembolic prophylaxis because of certain medical conditions (e.g., intracranial or active gastrointestinal bleeding). For these patients, the pharmacist can help to formulate a plan for the timing of initiation of appropriate thromboembolic prophylactic medications. The various types of prophylactic therapy include low-molecular-weight heparins, unfractionated heparin, sequential compression devices, and intravascular filters. Pharmacists must be familiar with the presenting clinical situation and should recommend the most appropriate method of prophylaxis on that basis.

\section{$\mathrm{H}$ is for Hyperactive or Hypoactive delirium}

Most critically ill patients experience some form of delirium during their ICU stay. ${ }^{8}$ Untreated delirium can lead to an increased length of stay in the ICU, as well as increases in costs, morbidity, and mortality. Tools such as the Intensive Care Delirium Screening Checklist" or the Confusion Assessment Method for the $\mathrm{ICU}^{10}$ can help the pharmacist to identify the presence of delirium. When delirium is diagnosed, a search for the cause (and for approaches to correct the cause) often relies on identifying potential drug-related causes of delirium. Treatments involving supportive and environmental measures and, in most cases, some form of pharmacological intervention with an antipsychotic drug may be considered. The pharmacist can provide guidance on the best choice of agent. Once an antipsychotic agent is chosen, the pharmacist should routinely assess whether the regimen and dose are optimal and should monitor the patient for efficacy and adverse reactions.

\section{$\mathbf{U}$ is for stress Ulcer prophylaxis}

Patients receiving mechanical ventilation in the ICU are at risk of stress ulcers, and appropriate prophylactic medications should be administered. ${ }^{11}$ The most commonly used agents for this indication include histamine receptor antagonists and proton pump inhibitors. The pharmacist should ensure that the patient is receiving a prophylactic agent and should also help to determine the most appropriate agent, according to the patient's medical history. Once the patient's condition improves and there is no longer a risk of stress ulcers, the pharmacist should reassess whether the prophylactic agent can be discontinued.

\section{G is for Glucose control}

Blood glucose control is an important aspect of patient care in the ICU. The pharmacist can play a significant role in maintaining blood glucose within the patient's target range by helping to select the most appropriate pharmacological regimen from the wide variety of insulin regimens or oral hypoglycemic drugs that are available. The pharmacist can also help to titrate the doses of these agents and reassess for efficacy and safety on a regular basis. For a number of reasons, the blood glucose concentration of an ICU patient may fluctuate, and the pharmacist can help to identify drug-related causes such as glucocorticoids, propofol, and atypical antipsychotics. ${ }^{12}$

\section{$M$ is for Medication reconciliation}

Medication reconciliation is an important aspect of care for any patient admitted to the hospital. This process involves reviewing medications that the patient was receiving before admission and deciding which drugs need to be restarted to 
ensure continuity of care. Ideally, medication reconciliation is performed upon admission to any patient care unit, with each transfer of care (either level or location), and before discharge. ${ }^{13}$ The pharmacist plays an integral role in this process, which should be performed routinely. For many patients, medications taken before admission are not restarted because acute medical conditions preclude their use. Another important issue is identifying discontinued medications for which there is a high risk of experiencing withdrawal symptoms (e.g., benzodiazepines and selective serotonin reuptake inhibitors).$^{14}$ Typically, these medications should be restarted as soon as possible to reduce complications.

In the ICU setting, it is often difficult to obtain information about patients' preadmission medications because patients may be sedated, confused, or unable to verbalize. The pharmacist must therefore rely on various other means of obtaining this information. Potential sources of information include the patient's family members, physician, community pharmacy, old medical records, electronic health records, and visual inspection of pill bottles. Information must be considered for all types of medications used by the patient, including prescription medications, nonprescription products, herbal and homeopathic medications, vaccines, vitamins, drug samples, and even illicit drugs.

\section{A is for Antibiotics or Anti-infective agents}

Patients admitted to the ICU either have an infection already or are at increased risk of acquiring an infection during their stay. As such, pharmacists can play a crucial role in antimicrobial stewardship. Stewardship activities include selecting the optimal antimicrobial agent and de-escalating treatment once culture and susceptibility results are available. Factors such as medical history (e.g., comorbid renal or hepatic dysfunction), allergies, recent antibiotic use, and recent admission to a health care facility must be considered before a regimen is chosen. In addition, the efficacy and safety of the regimen should be monitored and the dosage or agents adjusted as needed. In most institutions, therapeutic drug monitoring of antibiotics is performed by pharmacists.

\section{I is for Indications for medications}

Given the complex nature of care in the ICU, it is not unusual for patients to be treated with a large number of medications, most of which are initiated during their ICU stay. The pharmacist should review all regularly scheduled and as-needed medications daily to ensure that each has an appropriate indication. Any medication that is no longer indicated should be discontinued, to reduce the risk of adverse events, drug interactions, medication errors, and cost.
Conversely, the pharmacist should assess whether the patient has an untreated indication for which drug therapy would be appropriate.

\section{$D$ is for drug Dosing}

In critically ill patients, renal and hepatic function may fluctuate frequently. The pharmacist is in an ideal position to suggest dose adjustments based on clinical parameters, including indicators of renal and hepatic function, to prevent accumulation of drugs while ensuring adequacy of doses to achieve desired clinical end points. It is also important that the pharmacist continually reassess and adjust doses as the patient's condition begins to improve, to prevent underdosing. Therapeutic drug monitoring may be required for some medications, and the pharmacist can provide such monitoring when appropriate.

\section{E is for Electrolytes, hematology, and other laboratory tests}

Blood work is a part of daily care for critically ill patients. Pharmacists should monitor patients for drug-related causes of abnormalities in electrolytes, hematology results, or other laboratory values and discuss treatment alternatives with the other members of the health care team. In addition, the pharmacist may recommend initiation or discontinuation of electrolyte supplements, nutrients, minerals, blood, and fluid products if appropriate.

\section{$\mathrm{N}$ is for No drug interactions, allergies, duplication, or side effects}

With the multitude of medications that a typical ICU patient is receiving, the risk of drug interactions increases. It is therefore important to identify clinically important potential and actual drug-drug, drug-food, and drug-laboratory interactions immediately and to recommend alternative therapy. In addition, if a patient appears allergic to a particular medication, the pharmacist should perform an assessment to determine if the reaction is a true medication allergy or an intolerance. Following the assessment, the pharmacist can assist in either recommending alternatives or monitoring for adverse drug reactions. The pharmacist should also look for duplication of medication therapy and stop unnecessary medications. Finally, the pharmacist should assess whether the patient is regularly experiencing adverse effects from a medication.

\section{$\mathbf{S}$ is for Stop dates}

Not all medications prescribed for a patient are meant to continue indefinitely. For example, drugs that may require a stop or reassessment date include (but are not limited to) corticosteroids and anti-infective agents. The pharmacist should 
discuss the appropriate duration of medications with other members of the health care team. Conversely, the pharmacist should ensure that medications are not discontinued prematurely.

\section{DISCUSSION}

The FASTHUG-MAIDENS mnemonic is the first published standardized, structured approach to identifying drug-related problems in the ICU. This mnemonic may help pharmacists in a number of ways. First, this standardized, structured approach to practice ensures that the essential aspects of pharmaceutical care are met consistently. Second, use of the mnemonic may reduce anxiety and apprehension among pharmacists not familiar with the ICU setting by providing a stepwise approach to identifying drug-related problems. Finally, the mnemonic is a good teaching tool for hospital pharmacy students and residents with little or no ICU experience. This mnemonic allows the student to see beyond the complexities of care for a typical ICU patient and to focus on identifying and resolving drug-related problems. The mnemonic can be readily implemented in practice. However, for students and pharmacists who do not routinely practise in the ICU, it is advisable to have knowledge of the common ICU concepts that will be encountered in the course of applying the mnemonic.

Although the FASTHUG-MAIDENS mnemonic focuses on drug-related problems in the ICU setting, we believe that it has applicability outside the ICU as well. Research should be conducted to assess the influence of the FASTHUGMAIDENS approach on patient outcomes.

\section{References}

1. Vincent JL. Give your patient a fast hug (at least) once a day. Crit Care Med 2005;33(6):1225-1229.

2. Papadimos TJ, Hensley SJ, Duggan JM, Khuder SA, Borst MJ, Fath JJ, et al. Implementation of the "FASTHUG" concept decreases the incidence of ventilator-associated pneumonia in a surgical intensive care unit Patient Saf Surg 2008;2:3.

3. Vincent WR 3rd, Hatton KW. Critically ill patients need "FAST HUGS BID” (an updated mnemonic). Crit Care Med 2009;37(7):2326-2327.

4. Jacobi J, Fraser GL, Coursin DB, Riker RR, Fontaine D, Wittbrodt ET, et al.; Task Force of the American College of Critical Care Medicine (ACCM) of the Society of Critical Care Medicine (SCCM), American Society of Health-System Pharmacists (ASHP), American College of Chest Physicians. Clinical practice guidelines for the sustained use of sedatives and analgesics in the critically ill adult. Crit Care Med 2002; 30(1):119-141.

5. Kress JP, Pohlman AS, O'Connor MF, Hall JB. Daily interruption of sedative infusions in critically ill patients undergoing mechanical ventilation. N Engl J Med 2000;342(20):1471-1477.
6. Carson SS, Kress JP, Rodgers JE, Vinayak A, Campbell-Bright S, Levitt J, et al. A randomized trial of intermittent lorazepam versus propofol with daily interruption in mechanically vented patients. Crit Care Med 2006;34(5):1326-1332.

7. Geerts WH, Bergqvist D, Pineo GR, Heit JA, Samama CM, Lassen MR, et al.; American College of Chest Physicians. Prevention of venous thromboembolism: American College of Chest Physicians evidence-based clinical practice guidelines (8th edition). Chest 2008;133(6 Suppl): 381S-453S.

8. Pun BT, Ely EW. The importance of diagnosing and managing ICU delirium. Chest 2007;132(2):624-636.

9. Bergeron N, Dubois MJ, Dumont M, Dial S, Skrobik Y. Intensive care delirium screening checklist: evaluation of a new screening tool. Intensive Care Med 2001;27(5):859-864

10. Ely EW, Inouye SK, Bernard GR, Gordon S, Francis J, May L, et al. Delirium in mechanically ventilated patients: validity and reliability of the confusion assessment method for the intensive care unit (CAM-ICU). JAMA 2001;286(21):2703-2710.

11. Daley RJ, Rebuck JA, Welage LS, Rogers FB. Prevention of stress ulceration: current trends in critical care. Crit Care Med 2004;32(10): 2008-2013.

12. Anger KE, Szumita PM. Barriers to glucose control in the intensive care unit. Pharmacotherapy 2006;26(2):214-228.

13. Pronovost P, Weast B, Schwarz M, Wyskiel RM, Prow D, Milanovich SN, et al. Medication reconciliation: a practical tool to reduce the risk of medication errors. J Crit Care 2003;18(4):201-205.

14. Black K, Shea C, Dursun S, Kutcher S. Selective serotonin reuptake inhibitor discontinuation syndrome: proposed diagnostic criteria. $J$ Psychiatry Neurosci 2000;25(3):255-261.

Vincent H Mabasa, BSC(Pharm), ACPR, PharmD, is Clinical Coordinator with Lower Mainland Pharmacy Services and Clinical Associate Professor, Faculty of Pharmaceutical Sciences, The University of British Columbia, Vancouver, British Columbia.

Douglas L Malyuk, BSC(Pharm), ACPR, PharmD, is a Clinical Pharmacy Specialist-Intensive Care with Royal Columbian Hospital, New Westminster, British Columbia.

Elisa-Marie Weatherby, BSC(Pharm), ACPR, PharmD, is a Clinical Pharmacist with Peace Arch Hospital, White Rock, British Columbia.

Alice Chan, BSc(Pharm), ACPR, PharmD, is Clinical Practice Leader, Alberta Health Services, and Clinical Adjunct Professor, Faculty of Pharmacy and Pharmaceutical Sciences, University of Alberta, Edmonton, Alberta.

\section{Address correspondence to:}

Dr Vincent $\mathrm{H}$ Mabasa

Lower Mainland Pharmacy Services

Burnaby Hospital

3935 Kincaid Street

Burnaby BC V5G $2 \times 6$

e-mail: Vincent.mabasa@fraserhealth.ca 